\title{
Desterreichische
}

\section{Botanische Zeitschrift.}

Die österreichische

botanische Zeitschrift erscheint

den Ersten jeden Monats.

Man pranumerirtauf selbe mit 8 t. ost. W. (16 R. Mark)

ganzjabrig, oder mit

ff. ost. W. (s R.Mark) hal bjahrig.

Inserate

die ganze Petitzeile

$15 \mathrm{kr}$. ost. W.
Organ

fur

Botanik und Botaniker.

No. 11.
Bxemplare

die frei durch die Post he. zogen werden sollen, sind blos bej der Redaktion (V. Bez., Schlossgasie Nr: 15) za prthumeriren. Im Wege des

Bachhandels ubernimmt Pränumeration

c. Gorold's Sohn in Wien.

sowie alle übrigen Buchhandlungen.

XXXII. Jahrgang.

WIEN.

November 1882.

TATEAT: Kalkfreie Cystolithen. Von Dr. Molisch. - Mymecodia echinata. Von Anteine. Bewegungsvermögen der Pfanzen. Von Tomasehek. - Neue Ascomyceten. Von Voss. - Inflorescentia foliosa. Von Dr. Borbás. - Zur Pressburger Flora. Von Sabransky. - Aus dem Küstenlande. Von Dr. Solla, - Nachtrage. Von Feh lner. - Cypern und seine Flora. Von Sintenis. - Flora des Etna. Von Strobl. - Literaturberichte. - Correspondenz. Von Keller, Blocki, Dr. Borbás, Sabransky, Scheppig. - Personalnotizen. - Vereine, Anstalten, Unternehmargen. -- Botanischer Tauschverein. - Zar Nachricht.

\section{Kleinere Arbeiten des pflanzenphysiolog. Institutes der Wiener Universität.}

$\mathrm{XV}$.

\section{Ueber kalkfreie Cystolithen.}

\section{Von Dr. Hans Molisch.}

Gelegentlich einer anatomischen Untersuchung der Goldfussia isophylla Nees fand ich in eigenthümlich gestalteten Markzellen Gebilde, die zwar mit den in der Rinde auftretenden Cystolithen ${ }^{1}$ ) grosse Aehnlichkeit hatten, sich aber von den letzteren hauptsächlich dadurch unterschieden, dass in denselben kein kohlensaurer Kalk abgelagert war.

Die Hauptmasse des Markes bei der genannten Goldfussia besteht aus dünnwandigen kurz-prismatischen Parenchymzellen; zwischen denselben eingestreut erscheinen jedoch ungemein dickwandige polyedrisch oder cylindrisch gestaltete Sklerenchymzellen, welche nicht selten die Länge eines Millimeters erreichen. Beinahe in jeder dieser idioblastisch ausgebildeten Markzellen findet man einen spiessähnlichen Cystolithen, der des $\mathrm{CaCO}_{3}$ vollständig entbehrt. Er durch-

1) Von einer Beschreibung derselben glaube ich absehen zu können, da sich eine solche in $\mathrm{K}$. Richter's Abhandlung: "Beiträge zur genaueren Kenntniss der Cystolithen und einiger verwandter Bildungen im Pfanzenreiche". LXXVI. Band der Sitzungsber. der k. Akad. d. Wissensch. I. Abth. Juli-Heft, Jahrg. 1877, p. 7, 11, 22, vorfindet.

0esterr. botan. Zeituchrift. 11. Heft 1882 . 
setzt entweder das ganze Lumen oder nur einen Theil desselben; im letzteren Falle endet er immer zugespitzt. Mitunter stossen die Cystolithen mehrerer übereinander liegender Zellen aufeinander, verschmelzen und bilden anscheinend einen einzigen auffallend langen Cystolithen, der die Querwände der Zellen durchsetzt.

Eine wichtige Eigenthümlichkeit, welche die kalkfreien Cystolithen der Goldfussia isophylla auszeichnet, und die allen anderen bis jetzt bekannt gewordenen Cystolithen fehlt, besteht darin, dass sie in der Regel mittelst mehrerer Stiele an die Zellwand befestigt sind. Am Längsschnitte gewahrt man an der Basis des Cystolithen gewöhnlich 1-2, seltener 3 Stiele und weiter an den Seiten bis 8, ja noch mehr. Sie sind sämmtlich kurz, mitunter an ihren Euden verbreitert und sowohl am Längsschnitt als am Querschnitt leicht aufzufinden.

Obwohl die kalkfreien Cystolithen in ihrer Form den normalen der Rinde ähneln, so unterscheiden sie sich doch schon äusserlich von den letzteren, indem ihre Oberfläche von mehr oder minder langen oft wellig verlaufenden Linien durehzogen scheint, und jenes warzige, höckerige Relief, wie man dasselbe an den Cystolithen der Acanthaceen und Urticeen zu sehen gewohnt ist, immer vermissen lassen. Auch die Schichtung und radiäre Streifung, welche auf dem Querschnitte der normalen Cystolithen so dentlich hervortreten, sind nicht zu sehen, doch erscheint die letztere bei vielen deutlich, wofern man Chromsäure kurze Zeit einwirken lässt.

Merkwürdigerweise treten die langgestreckten Sklerenchymzellen mit den kalkfreien Cystolithen nur im schmalen Theile des Internodiums auf, im angeschwollenen Theile, im Knoten dagegen werden sie vollständig verdrängt durch die gewöhnlichen mit $\mathrm{CO}_{3} \mathrm{Ca}$ versehenen Cystolithen ${ }^{1}$ ), welche stets in dünnwandigen Parenchymzellen eingebettet erscheinen.

Fertigt man aus dem Marke der Goldfussia Längsschnitte an, bedeckt dieselben mit dem Deckglas und lässt Salzsäure hinzufliessen, so findet kein Aufbrausen statt, nicht die kleinste Blase wird bei mikroskopischer Betrachtung wahrgenommen. Es könnte der Einwand erhoben werden, dass vielleicht doch $\mathrm{CO}_{2}$ sich entwickle, dass diese aber sofort von der Flüssigkeit absorbirt werde, allein dieser Einwand ist unberechtigt, da selbst bei Anwendung von sehr concentrirter Säure - wo also die Entwicklung der $\mathrm{CO}_{2}$ gewiss eine sehr energische wäre - nicht das geringste Bläschen entsteht. Dazu kommt noch, dass auch bei Anwendung von $\mathrm{SO}_{4} \mathrm{H}_{2}$ weder ein Aufbrausen, noch die Entstehung von Gypsnadeln, verfolgt werden kann. Berücksichtigt man schliesslich noch, dass die Cystolithen nach der Veraschung am Platinblech kein Skelet hinterlassen, so ist der Schluss berechtigt, dass $\mathrm{CaCO}_{3}$ in denselben nicht vorhanrlen, und dass auch

1) Dieselben sind von K. Richter übersehen worden, da er (l. c. p. 27) ausdrücklich hervorhebt, dass Cystolithen im Marke von Goldfussia isorhylla vollständig fehlen. 
sonst keine mineralische Substanz in erheblicher Menge abgelagert ist. Woraus bestehen die oben geschilderten Cystolithen also? Da sie sich auf Zusatz von Phloroglucin ${ }^{1}$ ) und Salzsäure schwach röthlich und nach vorbergehender kurz andauernder Behandlung mit Chromsäure auf Zusatz von Chlorzinkjod tief violett färben, so können wir dio gestellte Frage beantworten und sagen: sie bestehen aus schwach verholzter Cellulose.

Die beschriebenen Cystolithen, die, obwohl ungemein auffallend und charakteristisch, bis auf den heutigen Tag übersehen wurden, sind nicht etwa pathologischer Natur und vielleicht nur kranken Pflanzen eigenthümlich. Ich habe sechs gesunde und üppig wachsende Goldfussia isophylla Nees untersucht und bei allen obige Cystolithen gefunden. Sie sind daher nicht gut mit jenen kalkfreien Cystolithen zu vergleichen, welche $P$. Melnikoff ${ }^{2}$ ) in manchen Blättern von Ficus elastica und $\boldsymbol{F}$. australis hie und da zwischen normalen entdeckt hat; denn bei unserer Pflanze fehlen sie nie und liegen überdiess in idioblastisch entwickelten Sklerenchymzellen und zwar nur in solchen.

Dasselbe, was bezüglich der Cystolithen von Goldfussia isophylla Neesi) gesagt wurde, gilt auch von denen der $G$. glomerata Nees und Ruellia ochroleuca, da auch diese Pflanzen im Marke kalkfreie Cystolithen führen.

Wieso es nun kommt, dass in denselben kein $\mathrm{Ca} \mathrm{CO}$ abgelagert wird - ob die Zellmembran der betreffenden Zellen für kohlensauren Kalk impermeabel, oder ob die Säuren der Nachbarzellen eindringen und den Kalk in Lösung erhalten - diess zu entscheiden ist wohl heute unmöglich.

-

\section{Myrmecodia echinata Gaud.}

\section{Eine Ameisenpflanze von den Molukken.}

\section{Von Franz Antoine.}

(Mit einer lithogr. Tafel.)

Ein Epiphyt, welcher einen Knollen bildet, dessen Wurzeln die Aeste der Bäume umklammern und oben aus einem halsähnlichen Fortsatze in mehrere vierseitige, am Ende büschelweise beblätterte Zweige ausläuft.

Die Wurzeln entspringen seitlich an der Basis des Knollens, sie sind zahlreich, 5-10 Mm. dick, über $50 \mathrm{Cm}$. lang, an der Astrinde angepresst, hierdurch oft etwas verflacht, verzweigt, weit hin-

1) Vergleiche Wiesner: "Note über das Verhalten des Phloroglucins und einiger verwandter Körper zur verholzten Zellmembran". 77. Band der Sitzungsber. der k. Akad. d. Wiss. I. Abth. Jännerheft 1878.

$\left.{ }^{2}\right)$ Untersuchungen über das Vorkommen des $\mathrm{Ca} \mathrm{CO}_{3}$ in Pflanzen. Inaugural-Dissertation, Bonn 187\%, p. 35, 37 .

3) Goldfussia anisophylla Nees konnte ich leider nicht untersuchen. 